\title{
TRANSIENT ANALYSIS OF THE TRAPATT MODE IN AVALANCHE DIODES $\dagger$
}

\author{
M. KHOCHNEVIS-RAD, $\ddagger$ R. J. LOMAX and G. I. HADDAD \\ Electron Physics Laboratory, Department of Electrical and Computer Engineerıng, The University of Michigan, \\ Ann Arbor, MI 48109, U.S.A.
}

(Received 24 March 1978)

\begin{abstract}
The start-up transient behavior of the TRAPATT mode of oscullation in both $n^{+} p p^{+}$and $p^{+} n n^{+} S_{1}$ avalanche diodes is studied by computer simulation in the time domain through a device-circuit interaction program. The start-up transient is investigated for various rise times of the applied bias pulse The TRAPATT waveforms obtained from the simulation are in agreement with those observed experımentally. The theory of TRAPATT operation in a coaxial curcuit is revised and its previous inconsistencies are resolved. The lowfrequency bias-circuit oscillation is discussed and its relation to device instabilities and tuning-induced burnout are presented.
\end{abstract}

\section{INTRODUCTION}

The TRAPATT mode of oscillation in an avalanche diode is a large-signal phenomenon. The device is highly nonlinear when it is operated in this multifrequency mode and it is almost impossible to design a circuit $a$ priori which will sustain this mode of oscillation without critical adjustment. It is the objective of this paper to investigate by computer simulation how this large-signal operation is initiated and how the circuit is involved with a view to improving the understanding of circuit requirements. The major difficulty in this simulation is in obtaining a converged periodic solution for the devicecircuit interaction. Several prior computer simulations have been made. Transient simulation of the devicecircuit interaction was carried out by several authors [13]. In the calculations of Matsumura and Abe[1], a coaxial line with a voltage source in series with a resistive load was used, but fringing capacitances, package parasitics and the bias capacitor were not included. The simulation was performed in the time domain. Objections to this approach are that dc power is dissipated in the load, the effect of the package parameters cannot be determined, and the d.c. current through the device cannot be specified a priori. Attempts to remove some of these restrictions give rise to instabilities in simulation. Evans and Scharfetter [2] did an analysis in the time domain for the device and in the frequency domain for the circuit. Truncation of the lower limit of the integral used in the transformation between them in effect introduced a highpass filter which may have suppressed the low-frequency bias oscillation. In addition, complete details of the startup transient were not given. Bogan and Frey[3] used a current source in parallel with the resistive load but also neglected the d.c. blocking capacitor, fringing capacitances, and package parasitics. In this approach at the start of the interaction they had to apply an unrealistically large and short rise time bias pulse to initiate

†Supported by the U.S. Army Research Office.

$\ddagger$ Present address: Atomic Energy Commission, Tehran, Iran the oscillation across the device. As the oscillation started to grow, the voltage across the device decreased, due to the rectification in the device, and the current increased. The current had to be decreased in order to avoid the growth of the instability. their simulation was carried out for a time shorter than the transient of their circuit and therefore some doubt exists as to the significance of their results.

In this paper a brief description of the device and the circuit is given. The $p$-type $\mathrm{Si}$ diode and the coaxial circuit simulated are those of an experimental oscillator operated in our laboratory. The results of the devicecircuit interaction simulation are analyzed and discussed. Details of the transient analysis are given elsewhere [4].

\section{DEVICE CONSIDERATIONS}

The device consists of a $2-\mu \mathrm{m} p$-type Si diode with a doping density of $4 \times 10^{15} / \mathrm{cm}^{3}$ in the epitaxial region. The transport behavior of electrons and holes in a $p-n$ junction under nonequilibrium conditions is given by the current continuity equations. The one-dimensional continuity equation for holes [5] is

$$
-\frac{\partial J_{p}}{\partial x}+g_{p}-\frac{p}{\tau_{p}}=\frac{\partial p}{\partial t}
$$

where $g_{p}$ is the generation rate, $p$ is the local concentration, and $\tau_{p}$ is the lifetime of holes. The current flow of electrons and holes in the semiconductor is determined from the Boltzmann transport equation. With the usual approximation, the hole current can be written as

$$
J_{p}=-D_{p} \frac{\partial p}{\partial x}+q p v_{p}(E)
$$

The relation between carrier space charge and the electric field is given by Poisson's equation

$$
\frac{\mathrm{d} E}{\mathrm{~d} x}=\frac{q}{\epsilon}\left(N_{D}^{+}-N_{A}^{-}+p-n\right) .
$$


The finite-difference method is used to solve these nonlınear partial differential equations. The device program is written from the point of view of computational efficiency and is described by Bauhahn[6].

\section{CIRCUIT CONSIDERATIONS}

The curcuit model shown in Fig 1 consists of a lumped package equivalent curcuit together with a distributed transmission line. The circuit is termınated by a resistıve load For a realistic simulation, a blockıng capacitor must be present in the bias network to avoid dc power dissipation in the load. The experimental bias tee also Includes a series inductor which is omitted in the model because an ideal current source is assumed. The $50 \Omega$ lossless coaxial au line is $15 \mathrm{~cm}$ long and contains movable tuning slugs which are in direct contact with the inner face of the outer conductor Three of them serve as a low-pass filter while the fourth one, which is close to the diode, acts mainly as a capacitor TEM-mode propagation is assumed in the coaxial line which is realistic for the frequencies of interest The voltage and current at time $t$ at a point $x$ in the curcuit can be written as

$$
V(x, t)=f^{+}(x, t)+f^{-1}(x, t)
$$

and

$$
I(x, t)=y\left[f^{+}(x, t)-f^{-}(x, t)\right],
$$

where $f^{+}(x, t)$ is the forward voltage wave, $f^{-}(x, t)$ is the backward voltage wave, and $y$ is the characteristic admittance of the line at point $x \cdot f^{+}(x, t)$ and $f^{-}(x, t)$ are traveling voltage waves comprised of all frequencies present in the circuit If the voltage wave traveling toward a tuning slug discontinuity is known, then the reflected voltage can be found by matching the boundary conditions, 1.e. enforcing continuty of current and voltage. The expressions for the reflected voltage from the load plane, package plane, and tuning slug discontinuities are given elsewhere[4]. The package equivalent circuit adopted for this analysis is indicated in Fig. 2 in which $C_{p d c}$ is the sum of the device depletionlayer capacitance and any extra capacitance near the diode

In the computer program the coaxial line, which is $15 \mathrm{~cm}$ long, is divided into 600 space steps ensuring a very accurate calculation of voltage and current along the line This fine division of the transmission line is necessary in

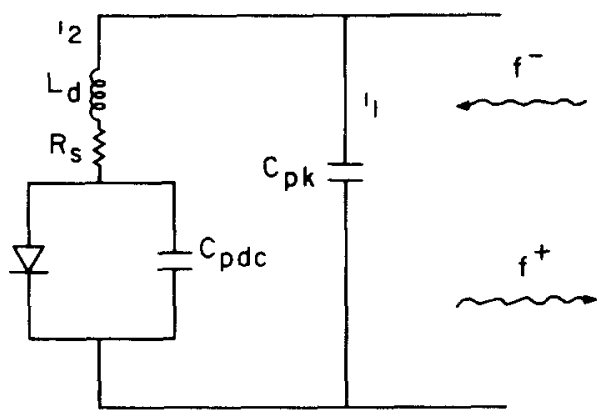

Fig 2 Equivalent circuit of the diode and package

order to reproduce accurately the higher harmonics which are present in the TRAPATT operation. The 600 values of the forward wave are stored in an array and the backward wave is treated similarly. The computer program has been written from the viewpoint of computational efficiency in the handling of these arrays In a time advancement $\Delta t$, the values of the forward and backward waves do not change between the discontinuties, because the transmission line is assumed to be lossless, and therefore they have only to be shifted in space by $\Delta x=c \Delta t$, where $c$ is the wave velocity (velocity of light). At the discontinuties, new values of the forward and backward waves are calculated. At each time step 1200 operations are required, 1184 shifting and 16 computing. For a transient which can last as long as $50 \mathrm{nsec}$ the corresponding $71,040,000$ shifting operations become quite expensive One way of avoiding this shifting is to change the frame of reference. In this system of reference the discontınuities move and the wave remains fixed in space. The discontinuities corresponding to the backward wave move to the right and those corresponding to the forward wave move to the left. Two pointer arrays store the positions of the discontinuities in the coaxial line at each time step and as a discontinuity arrives at the end of the array it is transferred to the beginning The technique just described decreases the cost of simulation by more than 100 times and makes feasible an extensive study of the circuit and the devicecurcuit interaction.

\section{DEVICE-CIRCUIT INTERACTION}

The simulation of the transient behavior of the TRAPATT mode is carried out by repeatedly utilizing

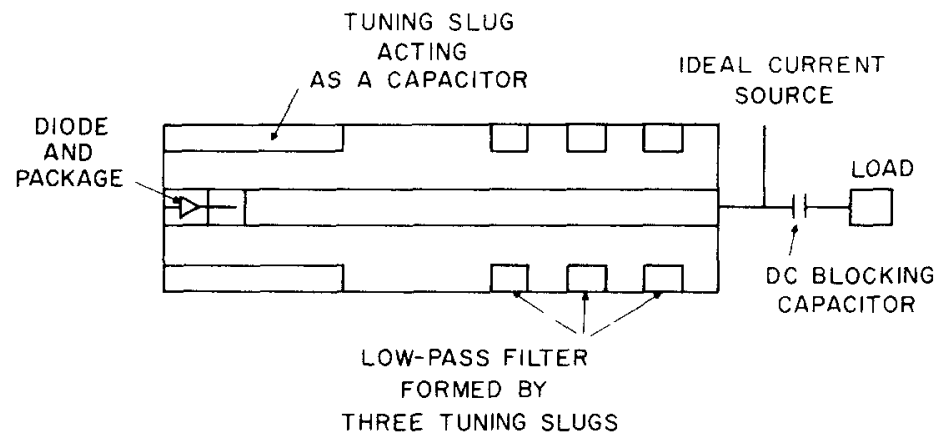

Fig 1 TRAPATT circuit used in the simulation 
the device simulation program which computes the current change due to a voltage change at the device terminals and the circuit simulation program which computes the response of the circuit voltage to the change in the device current. The device and the circuit parameters are those of an experimental TRAPATT oscillator which were measured very carefully and were used in the computer program. Due to the presence of high frequencies in the TRAPATT mode and its transient and also because of the long memory of the device and the circuit compared to one TRAPATT period, special care is needed to minimize the errors introduced by the interaction of the two finite-difference-method programs [4]. Parameters such as operating voltage, efficiency, and frequency are obtained from a selfconsistent solution of the device-circuit interaction. Because of the approximations made in the formulation of the device and circuit, and also because of inevitable small errors made in measuring the device and the circuit parameters, it is necessary to tune the circuit to obtain stable TRAPATT operation. The circuit may be tuned, as is done experimentally by changing the tuning slug positions in the coaxial circuit, but the direction in which the tuning slugs should be moved and by how many space steps are not well known. The circuit impedance that the diode needs to see is not known a priori, but it can be anticipated that the circuit impedance at the device plane should have a larger resistive part at the TRAPATT fundamental frequency than at the higher harmonics, and that the reactive part should be smaller. Under these conditions a large current can flow at the fundamental frequency and be dissipated in the load resistor while the energy at the higher harmonics can be stored in the circuit with little loss and can provide the overvoltage at each TRAPATT cycle. One way of avoiding this complicated method of tuning the circuit is to use the package parasitics to tune the circuit. This method has a tremendous advantage over the former since once the circuit impedance at the device plane is calculated, any changes in the package lead inductance or capacitance has an obvious effect on the circuit impedance at the device plane. Furthermore, there are now only two tuning parameters instead of three.

\section{TURN-ON TRANSIENT OF THE TRAPATT MODE UNDER A BIAS PULSE OF LONG RISE TIME}

The turn-on transient under a 4.3 nsec rise-time pulse is studied here. Longer rise times than this do not appreciably alter the behavior but increase the cost of simulation. The bias pulse which rises linearly to a final value of $12,000 \mathrm{~A} / \mathrm{cm}^{2}$ is applied to the diode through the coaxial line. To save computer time without significantly changing the results, the $2-\mu \mathrm{m}, p$-type diode is initially reverse biased at $10 \mathrm{~V}$. The voltage at the device terminals is shown in Fig. 3. The applied current pulse first drives the diode to avalanche breakdown $(\sim 2.75 \mathrm{nsec})$ and then the diode begins a rapid oscillation at a frequency around $11 \mathrm{GHz}$. These oscillations are essentially in the IMPATT mode. The circuit impedance at this frequency has a small resistive part and a large

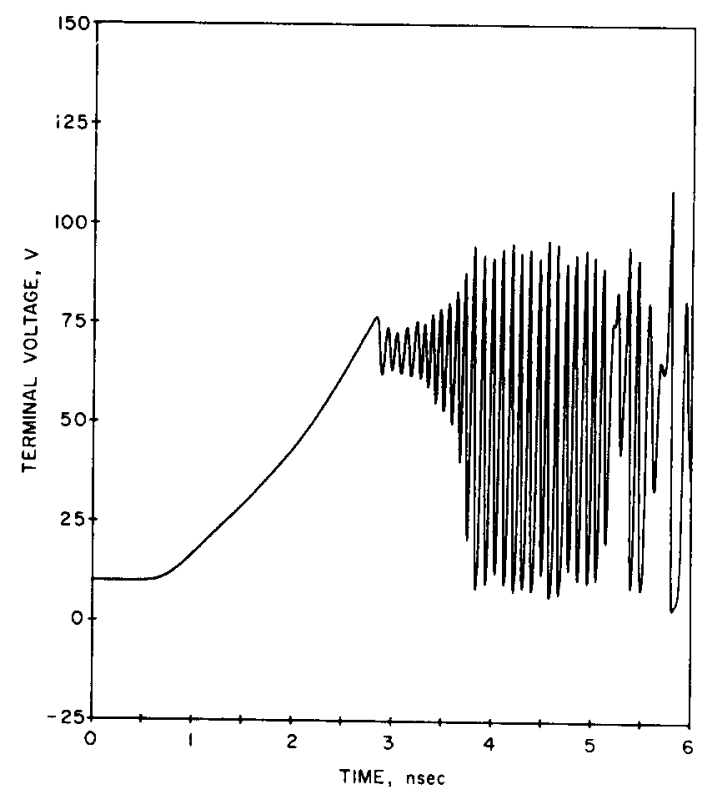

Fig. 3 Diode terminal-voltage response to a 4.3-nsec rise-tıme pulse

reactive (inductive) part. The IMPATT mode leads to a relaxation oscillation at the same frequency. The highfrequency oscillation builds up for several reasons: (1) the current drive keeps increasing, (2) the reflected wave from the circuit arrives at the device plane in a proper phase to amplify the oscillation and (3) the $Q$ of the circuit is large at this frequency. These large amplitude oscillations pump energy into the circuit at a higher rate than can be dissipated. The power at the TRAPATT frequency appears from the beginning of the transient because this device has small-signal negative resistance at the fundamental TRAPATT frequency. The presence of the TRAPATT frequency can be detected more clearly at the load because the low-pass filter attenuates high frequencies but passes the fundamental TRAPATT frequency with almost no attenuation. During the IMPATT oscillation the device pumps energy into the circuit at high frequencies, the circuit interacts with the device, and the device converts some of this energy into energy at the TRAPATT frequency. The relaxation oscillation pumps more RF energy into the circuit at high frequencies, thus more power can be converted to the TRAPATT frequency. The stored energy at high frequencies in the circuit reaches a point such that the transition from the relaxation oscillation to the TRAPATT mode becomes possible, the relaxation oscillation dies out and the TRAPATT mode dominates. The transition period between the relaxation oscillation and the TRAPATT corresponds to a phase and amplitude readjustment of different frequency components. The shortest transition period observed has been $1 \mathrm{nsec}$, which corresponds to the time it takes a wave to travel twice the length of the circuit. The kind of parametric effects explained for initiating the TRAPATT mode under a slow rise-time pulse are not observed under a fast rise-time pulse as will be seen later. Figure 4 shows the induced current through the device during this $6 \mathrm{nsec}$ of 


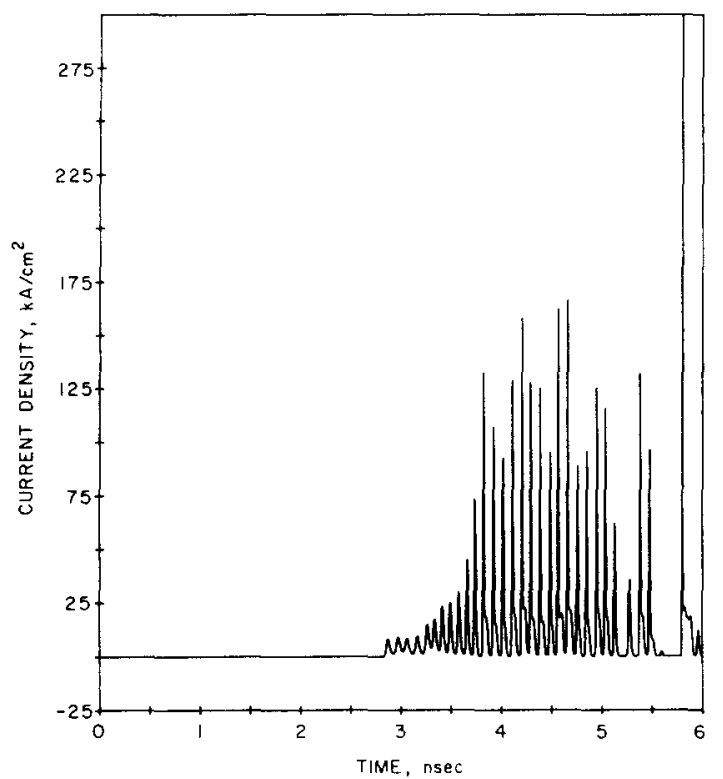

Fig 4. Diode particle current density response to a $43 \mathrm{nsec}$ rise-time pulse

the transient. The induced current, which has almost been zero prior to attainment of the breakdown voltage, starts to increase The spike in the induced current during the relaxation oscillation corresponds to the discharge of the lumped capacitors near the diode. It is important to note that during this $6 \mathrm{nsec}$ of simulation the device program has been called more than 15,000 times to ensure that all parameters change smoothly from one step to another.

\section{THE TRAPATT WAVEFORM AND THE OSCLLATOR THEORY}

The voltage at the device terminals after $49 \mathrm{nsec}$ of simulation is shown in Fig. 5, with two cycles being drawn on a large scale in Fig. 6. The waveform for a simplified model in which a square-wave current is assumed [7] is shown by the dashed line in Fig. 6. The simplified theory assumes that, at the second half-cycle, the voltage across the device is held constant and equal to the d.c. breakdown voltage. However the devicecircuit interaction simulation revealed that the voltage in the second half of the cycle (Fig. 5) does not recover to the breakdown voltage but oscillates at slightly above the dynamic d.c. voltage of the device. During the second half-cycle the current is almost entirely displacement current. This oscillation cannot be related to the IMPATT mode as was predicted by some authors, and is only due to the ringing of the circuit. The experimental TRAPATT voltage waveform reported by Yanai $e t$ al. [8] shows clearly that the voltage in the second half-cycle is significantly lower than the device d.c. breakdown voltage. This result should be expected because, when the device is operating in the TRAPATT mode, the average voltage across the device is usually one half of the d.c. breakdown voltage. If during the second half of the cycle the voltage is held at the d.c. breakdown voltage, the voltage in the first half-cycle should be zero,

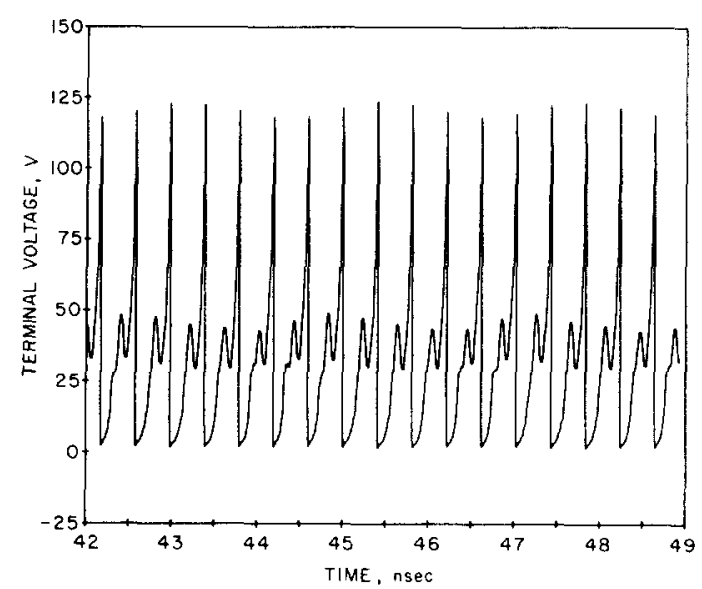

Fig 5 Voltage waveform of a p-type device operatıng in the TRAPATT mode (current density $=12,000 \mathrm{~A} / \mathrm{cm}^{2}$ )

which cannot be true. Consequently, in the second halfcycle the voltage has to be lower than the d.c. breakdown voltage. Due to the presence of a low-amplitude, low-frequency bias-circuit oscillation a completely stable TRAPATT waveform cannot be achieved and the TRAPATT waveform changes smoothly from one cycle to the next. It almost repeats itself every six cycles as can be seen in Fig. 5. The TRAPATT waveform was Fourier analyzed. At the fundamental frequency of $\sim 2.45 \mathrm{GHz}$ the device impedance is almost the conjugate of the circuit impedance. However, this is not true for the higher harmonics. The $Q$ of the circuit is low at the fundamental frequency and large at the higher harmonics which explains why the components at the fundamental frequency converge more rapidly than do the higher harmonics. A theory which has been proposed for the TRAPATT diode operating in a coaxial line is that the drop in diode voltage from breakdown to zero volts propagates down the transmission line and is reflected back by the low-pass filter with a reflection coefficient of almost minus one. When this reflected pulse reaches the diode, the diode voltage is close to breakdown. The pulse causes the voltage across the diode to increase to almost twice the breakdown voltage and the cycle repeats. However, when the diode is oscillating in the TRAPATT mode, the d.c. voltage across the diode and the entire line is almost half of the d.c. breakdown voltage. Therefore, the voltage pulse reflected from the low-pass filter must reverse its polarity around the dynamic d.c. voltage and not the static d.c. breakdown voltage. Furthermore, in the second half of the TRAPATT cycle the voltage across the device is significantly lower than the d.c. breakdown voltage. As a consequence, the reflected wave can at best drive the diode to its d.c. breakdown voltage and not to twice the breakdown voltage. In order to gain a better understanding of the operation of the oscillator, a movie was made which displays the voltage along the coaxial line as a function of time [9]. Figures 7 and 8 are taken from the movie. Figure 7 shows the state of the circuit at the start of a TRAPATT cycle where the device is biased at $50 \mathrm{~V}$; however, the voltage across the package is approximately $105 \mathrm{~V}$ as indicated in Fig. 7. 


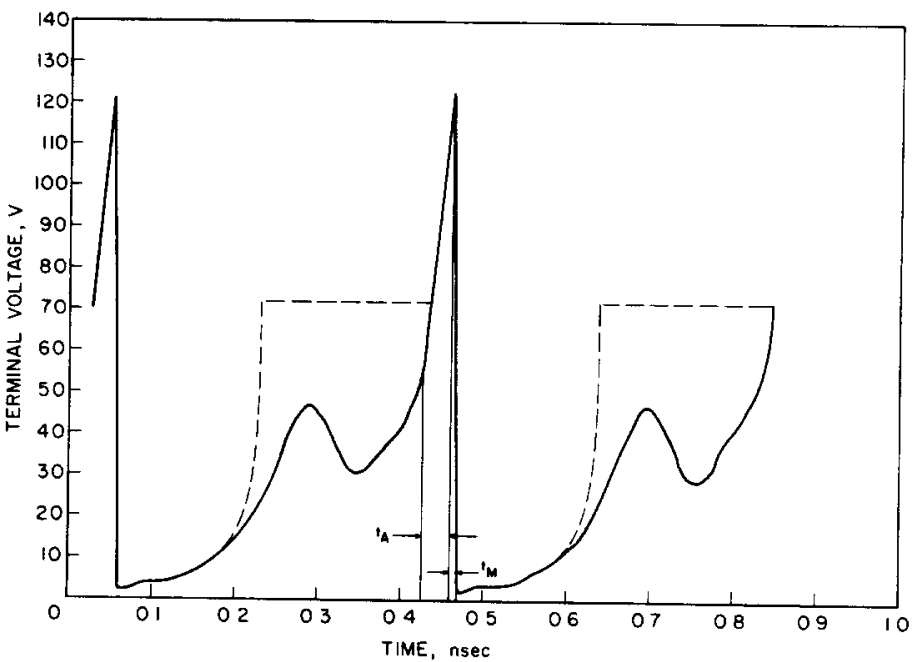

Fig. 6. $p$-type diode terminal voltage vs time after $17 \mathrm{nsec}$.

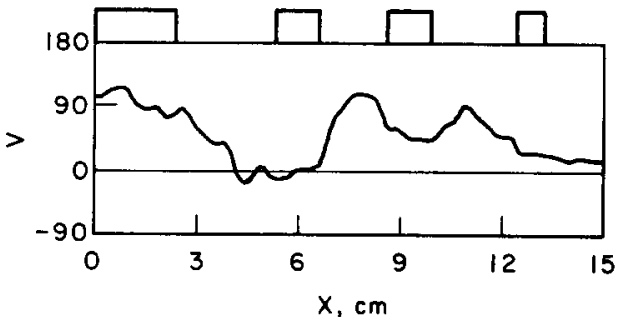

Fig. 7. State of the curcuit at the beginning of the TRAPATT cycle.

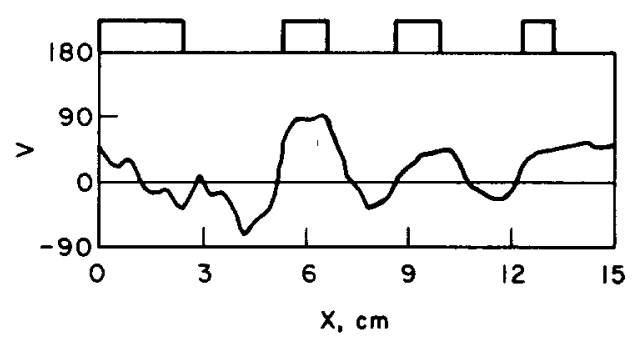

Fig. 8. State of the crrcuit at the half-period of the TRAPATT cycle.

The circuit voltage is zero at the first tuning slug of the low-pass filter. Figure 8 shows the voltage along the coaxial line at the midpoint of the TRAPATT cycle. The voltage across the first tuning slug of the low-pass filter is $100 \mathrm{~V}$ and the reflected wave from the diode is about to impinge on the first tuning slug. The preceding theory can be revised and its inconsistencies resolved as follows: when the voltage across the device collapses, the diode is in its highly conducting state and acts as a short across the circuit. Therefore at this stage the wave reflected from the device plane has almost the same magnitude as the incident wave but has reverse polarity. If $f_{d}{ }^{+}(t)$ represents the reflected voltage pulse from the device plane, the first tuning slug of the low-pass filter reaches its highest voltage when $f_{d}^{+}(t)$ arrives. The reflected wave from the plane of the low-pass filter can be calculated by writing the continuity of voltage at the plane of the low-pass filter:

$$
f_{d}^{+}(t)+f_{d}^{-}(t)=f^{+}(t)+f^{-}(t),
$$

where $f^{+}(t)+f^{-}(t)$ represents the total voltage across the first tuning slug, which is $100 \mathrm{~V}$ at this time of the cycle. The reflected voltage wave $f_{d}^{-}(t)$ is given by

$$
f_{d}^{-}(t)=100-f_{d}^{+}(t)
$$

but $f_{d}^{+}(t)$ is negative; therefore, $f_{d}^{-}(t)$ is very large and can easily cause the overvoltage across the device.

The frequency of TRAPATT operation is determined by the round-trip propagation time of the pulse between the reflecting plane of the filter and the diode and the delay time between the reflected and incident pulses on the packaged diode. The total period of the oscillation includes the charging time and the plasma formation time indicated by $t_{A}$ and $t_{M}$ in Fig. 6, respectively. $t_{A}$ and $t_{M}$ depend strongly on package parameters and, in particular, on the lead inductance. The period of the TRAPATT oscillation, $T=t_{C}+t_{A}+t_{M}+\tau_{p}$ (where $\tau_{p}$ is the total time delay in the package), gives the frequency of operation in the TRAPATT mode $(\sim 2.45 \mathrm{GHz})$. The TRAPATT frequency is not harmonically related to the starting IMPATT frequency.

\section{LOW-FRRQUENCY BIAS-CIRCUTT OSCILATION}

A low-frequency oscillation which modulates the amplitude of the TRAPATT oscillation can be seen in Fig. 5. This oscillation is related to the circuit as follows. If a steady-state TRAPATT oscillation is reached and the line is energized to the dynamic d.c. voltage $V_{E}$, a constant direct current then flows from the source to the diode and the voltage across the blocking capacitor is $V_{E}$. An increase in the average current through the device will cause the RF voltage amplitude to increase which, in turn, because of the rectification effect, will produce a decrease in the average voltage across the device. This decrease causes the line to discharge through the device and this information is transmitted to 


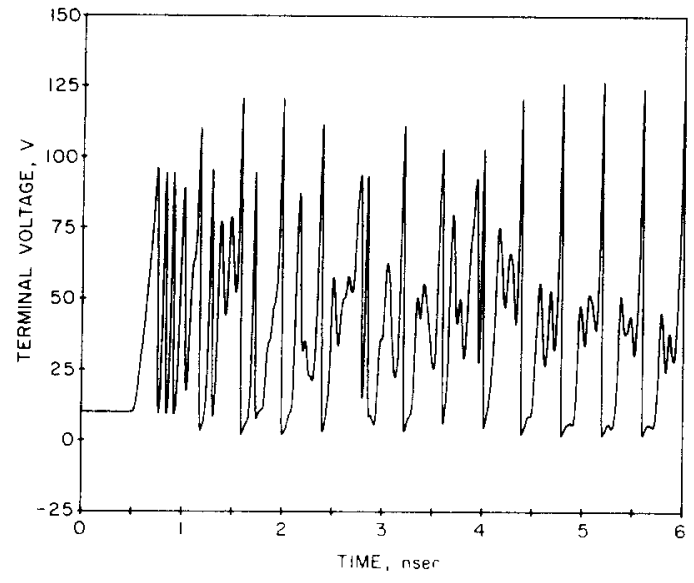

Fig 9. Start-up voltage transient of a p-type device under a square current pulse (current density $=12,000 \mathrm{~A} / \mathrm{cm}^{2}$ )

the load where the d.c. blocking capacitor also discharges. The RF voltage amplitude continues to build up until the capacitor and the line are no longer capable of providing the current required to sustain the higheramplitude oscillation. This point marks the half-period of the low-frequency cycle. From this point to the end of the cycle the RF voltage amplitude decreases while the average voltage increases. At the same time the average current through the device decreases because the current source is charging the line as well as the blocking capacitor. This process then repeats. The low-frequency oscillation is not harmonically related to the TRAPATT mode and depends on the circuit length and the d.c. blocking capacitor

\section{DEVICE-CIRCUIT INTERACTION UNDER VARIOUS PULSE RISE TIMES}

Due to the high power density at which the TRAPATT mode operates and the limited power dissipation of the device, the device must be operated under pulse bias conditions. Under pulse conditions, the turn-on time may become a significant fraction of the bias pulse width and therefore it is useful to study the start-up of the oscillator under current pulses of various rise times. The turn-on time can be defined as the time between the instant when the applied current pulse reaches the device and the instant when a steady-state oscillation is established. Pulses having longer rise tumes than $5 \mathrm{nsec}$ are not of interest since the turn-on time is then limited, mainly by the rise time of the pulse Pulses having much shorter rise times than $5 \mathrm{nsec}$ will drive the diode to large-signal operation immedrately, and the possibility exists of initrating the TRAPATT mode more quickly.

\section{TURN-ON TRANSIENT UNDER A SQUARE CURRENT PULSE}

Figure 9 shows the voltage transient obtained when a square current pulse of $12,000 \mathrm{~A} / \mathrm{cm}^{2}$ was applied to the diode through the coaxial line. The device starts with a large amplitude relaxation oscillation which lasts a time equal to one TRAPATT period. After the initial rise the voltage immediately collapses to less than $10 \mathrm{~V}$ and subsequently the reflected pulse produces an overvoltage across the device after a time equal to one TRAPATT cycle. The transition between the relaxation oscllation and the TRAPATT mode takes place in $3 \mathrm{nsec}$ The transition state is a mixed mode composed of both relaxation and TRAPATT oscillations. A low-frequency bias circuit oscillation is present The large amplitude modulation it produces at the start of the transient is detrimental to the establishment of a stable TRAPATT oscllation. This is because at its low-voltage swing the bias circuit oscillation causes the overvoltage to dimınısh and therefore to produce only a low-density plasma in the device. It takes only a short time for this plasma to be removed, which delays the establishment of a repet1tive pattern. The turn-on time of the oscillator is $35 \mathrm{nsec}$ when the square current pulse is applied. The turn-on tıme under 0.05 nsec pulse rise time was almost as long as the case of $4.3 \mathrm{nsec}$, but more power was avalable at the fundamental frequency earlier in the transient than in the 4.3 nsec case

\section{STUDY OF $n$-TYPE TRAPATT DEVICES UNDER 43 nsec PULSE RISE TIME}

Schroeder and Haddad[10] predicted that the $p^{+} p n^{+}$ Si diode is capable of better RF power performance and higher efficiency than the $p^{+} n n^{+}$structure when the device is operating in the IMPATT mode. However, it is not well established which diode will have better performance if both are operating in the TRAPATT mode. The most thorough investigation of the TRAPATT mode of oscillation in $\mathrm{S}$ l avalanche diodes was made by Lee [7] who used a square-current driven sımulation program His results indicate that the $p^{+} p n^{+}$device and its complementary structure have identical peak efficiency (of course, the differing lonization rates. mobility, etc of holes and electrons prevents complete complementarity) The preceding investigation contradicts the experimental results reported by Trew[11], indicatıng superior performance of the $p^{+} p n^{+}$diode over its complementary structure Care should be taken in interpreting experimental measurements because it is not easy to fabricate two identically complementary diodes. Furthermore, it is difficult to determine whether the circuit parameters are those which optimize TRAPATT operation in both structures. There are no clear guidelines as to how the circuit parameters should be changed in order to obtain the best TRAPATT performance. Concerning computer simulation, it can be argued that the same current drive was used to simulate the $p^{+} p n^{+}$device and its complementary structure and that the optimum voltage and current waveforms might be different for both devices Because of these difficulties, an exact comparison of these two structures if virtually impossible. In order to make a meaningful comparison between two complementary structures, both diodes were simulated in the same coaxıal circuit operatıng under the same bias current density. After stable TRAPATT operation had been obtained with a $p$-type dode, the device was replaced by its complementary structure while all the circuit, package, and device parameters remained unchanged 
except for the sign of the doping in the epitaxial region. In this case a coherent TRAPATT mode could not be achieved. To obtain stable $n$-type TRAPATT operation, the package lead inductance had to be decreased from 0.41 to $0.21 \mathrm{nH}$ but all other parameters were kept unchanged. This decrease was suggested by the higher ionization rate of electrons. Figure 10 shows that the oscillation starts with the IMPATT mode at a frequency of $13.8 \mathrm{GHz}$ but these oscillations decay after a few cycles. At that time a new IMPATT oscillation at a higher frequency $(15 \mathrm{GHz})$ starts and builds up very quickly to a large amplitude relaxation oscillation. This portion of the transient can be explained as follows: during the first portion of the transient, where the applied current pulse drives the diode from $10 \mathrm{~V}$ to avalanche breakdown, the device acts like a fixed capacitor and as a result this portion of the transient is similar to that of the complementary structure. The bias current drives the device voltage above its breakdown value and the device starts oscillating. The starting frequency of oscillation depends partly on the current through the device and partly on the impedance seen by the device. The device starts oscillating at $13.8 \mathrm{GHz}$, however, in order for the oscillation to build up, the $Q$ of the circuit should be large at that frequency. The calculated impedance seen by the diode looking into the circuit through the package shows that the $Q$ of the circuit is low at this frequency and therefore the oscillation cannot build up. These oscillations eventually die out because the bias current density keeps increasing [4]. A new IMPATT oscillation starts to build up at approximately $15 \mathrm{GHz}$. This higher IMPATT frequency is expected because at this stage in the transient the current through the device is large [4]. The oscillations at $15 \mathrm{GHz}$ build up because the circuit has a large $Q$ at this frequency. This IMPATT oscillation changes to a relaxation oscillation after a few cycles. The relaxation oscillation lasts for a shorter period of time than it does in the complementary structure, but the transition from relaxation oscillation to the TRAPATT mode takes longer because the $n$-type device is noisier than the $p$-type one [9]. After 9 nsec of simulation, the device switches into stable TRAPATT operation. The

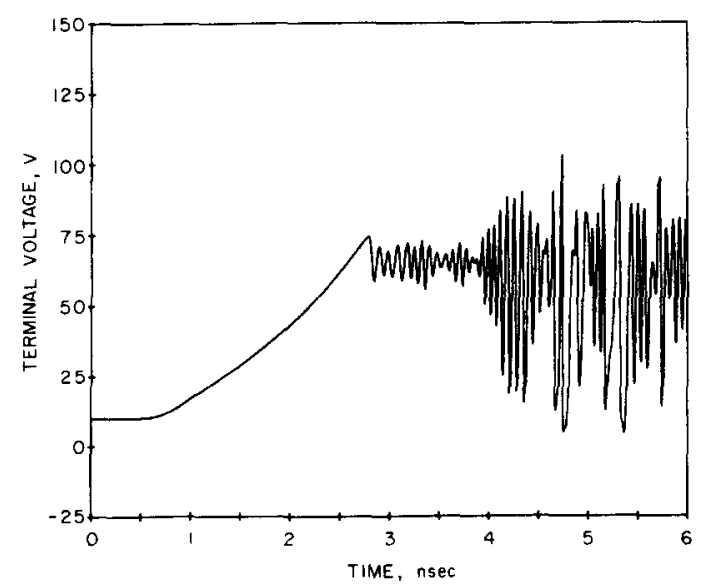

Fig. 10 Start-up voltage transient of an $n$-type device (current density $=12,000 \mathrm{~A} / \mathrm{cm}^{2}$, package lead inductance $=021 \mathrm{nH}$ ) $n$-type TRAPATT waveforms are different from those of the complementary structure. During the second halfcycle more ringing is present on the voltage waveform. Furthermore, the plasma extraction time is marked by a slight voltage recovery followed by a new collapse before the final removal of the plasma. The frequency of the TRAPATT oscillation is $2.54 \mathrm{GHz}$ instead of $2.45 \mathrm{GHz}$ obtained with the complementary structure. The higher frequency is due to the lower inductance of the package which causes the drop in voltage across the package and the overvoltage across the device to last a shorter time. The efficiency of operation at the fundamental frequency was lower than for the complementary structure, the voltage and current amplitude are very close to those of the $p$-type diode, and the lower efficiency is due to the smaller phase angle between the current and voltage.

\section{DEVICE INSTABILITY AND TUNING-INITIATED FAILURE}

Tuning-induced burnout has been observed in $\mathrm{Si}$ avalanche diode oscillators operating in the TRAPATT mode and its occurrence is more frequent in the $n$-type device than in the $p$-type one. In order to understand the mechanism which causes this failure, the device-circuit interaction was simulated for the following three cases: (1) a previously stable TRAPATT oscillator was mistuned, (2) the oscillator was tuned for a stable TRAPATT operation and (3) a stable TRAPATT oscillator was slightly mistuned. In the first case the output spectrum of the oscillator was extremely noisy but the amplitude of the low-frequency bias circuit oscillation was small. In the second case the low-frequency oscillation which amplitude modulates the TRAPATT oscillation was larger than in the previous case, and its maximum voltage swing occurred at the beginning of the transient. The amplitude of this low-frequency oscillation then decreases and eventually becomes constant. In the third case the amplitude of the bias circuit oscillation is significantly higher than in the other cases, particularly at the start of the TRAPATT oscillation. The voltage and current amplitudes, which arise due to bias circuit oscillation, would be large enough to cause burnout of an actual device. It has been observed experimentally as well as in computer simulation that, in TRAPATT operation, the $n$-type device is more sensitive to changes in the circuit parameters than its complementary structure. Because the $n$-type device requires more critical tuning for stable TRAPATT operation than the $p$-type one, bias circuit oscillations are more likely to build up to a destructive' level while the circuit is being tuned. This could explain why the $n$-type device is observed to have a higher failure rate than the $p$-type device

\section{CONCLUSIONS}

The start-up transient behavior of the TRAPATT mode of oscillation has been studied in both $p^{+} n n^{+}$and $n^{+} p p^{+}$Si avalanche diodes by means of computer simulation. The simulation was carried out in the time domain through the interaction of a device program and a pro- 
gram which accurately models the coaxial circuit. The study shows that, when excited by a bias pulse having a slow rise time $(4.3 \mathrm{nsec})$, the TRAPATT mode is triggered by initially an IMPATT oscillation, followed by a relaxation oscillation, which finally changes via a transition period to the TRAPATT mode. It was found that the voltage in the second half of the cycle did not recover to the breakdown voltage as has previously been suggested but oscillated around a value slightly above the dynamic d.c. voltage of the device. A revised theory of operation based on the circuit response was therefore given to explain the formation of the overvoltage which is required to trigger the next cycle.

Simulation of the $n$-type structure revealed that the frequency and duration of each mode of oscillation was different from those for the $p$-type structure and the TRAPATT waveform was slightly different, but the general behavior was very much the same. Lowfrequency bias circuit oscillations were present in both the $p$ - and $n$-type device simulations, but were much more difficult to reduce in the $n$-type device. As a result of this, these oscillations are much more likely to build up to a destructive level in the $n$-type device and cause tuning-induced burnout.

\section{RETERENCES}

1 M. Matsumura and H. Abe, IEEE Trans. Microwave Theory Tech (Correspondence) MTT-18, 975 (1970).

2 W. J Evans and D L. Scharfetter, IEEE Trans. Electron Dev. ED-17, 397 (1970)

3. Z. Bogan and J Frey, IEEE Trans. Electron Dev. ED-24, 128 (1977)

4. M Khochnevis-Rad, Transient Analysis of the TRAPATT Mode, Ph D Dissertation, The University of Michigan (1977)

5 In this paper equations for electrons are not given The equations are simular to those of holes with an appropriate sign change

6 P E. Bauhahn, Properties of Semiconductor Materials and Microwave Transit-Tume Devices, Ph.D. Dissertation. The University of Michigan (1977)

7 C. M. Lee, Properties of Avalanche Transit-Tume Devices, Ph D. Dissertation, The University of Michıgan (1974)

8 H. Yanai, H Torizuka, N. Yamada, and K Ohkubo, IEEE Trans. Electron Dev. ED-17, 1067 (1970).

9. M. Khochnevis-Rad, R J Lomax and G I Haddad In Tech Digest 1976 Int Electron Devices Meeting. pp 106-109, Washington, D C. (1976)

$10 \mathrm{~W}$ E. Schroeder and G I. Haddad, Proc IEEE (Correspondence) 59, 1245 (1971)

11. R Trew, Properties of $S$-Band TRAPATT Diode Oscillators $\mathrm{Ph}$ D Dissertation, The Unıversity of Michıgan (1975) 